\title{
SEVERE COVID-19 IN A SUSTAINED REMISSION LUPUS PATIENT - CASE REPORT
}

Sean Hideo Shirata Lanças ${ }^{1, \star}$, Matheus Zanata Brufatto', Laura Maria Silva de Siqueira', Douglas Squizatto Leite', Luiz Eduardo Valente', Fabio Vicente Leite', Henrique Pereira Sampaio', Andrea de Almeida Peduti Batista', Sula Glaucia Lage Drumond Pacheco'

1.Universidade Estadual Paulista, Botucatu (SP), Brazil.

*Corresponding author: sslancas@hotmail.com

\section{BACKGROUND}

The coronavirus disease 2019 (COVID-19) presents with a great heterogeneity, with studies showing some risk factors associated to worst outcomes. Its relationship with autoimmune diseases and immunosuppressant drugs is still not clear. We presented a case of a young female patient with systemic lupus erythematosus (SLE) that was in a sustained clinical remission, with COVID-19 diagnosis evolving to a severe form with the need for invasive ventilation; however, evolving with recovery and hospital discharge.

\section{CASE REPORT}

F.L.A., 37 years old, female, followed in our ambulatory with SLE diagnosis since she was 15, with a cutaneous and articular predominant form, without severe activities, recently in sustained remission for more than one year, and in a regular use of hydroxychloroquine without corticosteroids. She was admitted at our hospital with a prior history of one-week flu syndrome progressing with persistent fever, dyspnea and desaturation, demanding hospitalization and oxygen supplementation. She had contact with a previous confirmed COVID-19 case, and had no organ dysfunctions nor clinical or laboratory lupus activities. Complementary exam showed leukocytosis and lymphopenia, high PCR and D dimer. Lungs computed tomography (CT) scan compatible with $>50 \%$ affected parenchyma (Fig. 1), besides a positive RT-PCR swab for severe acute respiratory syndrome coronavirus 2 (SARS-CoV-2). On the second day, she evolved with worsening hypoxemia and need for orotracheal intubation and invasive ventilation, being transferred for the critical care unit, receiving protocol treatment including neuromuscular blocking agents and prone position ventilation. She was also given ceftriaxone, azithromycin and dexamethasone. Showing a progressive clinical improvement, she was extubated within 6 days and had hospital discharge after 14 days of hospitalization.

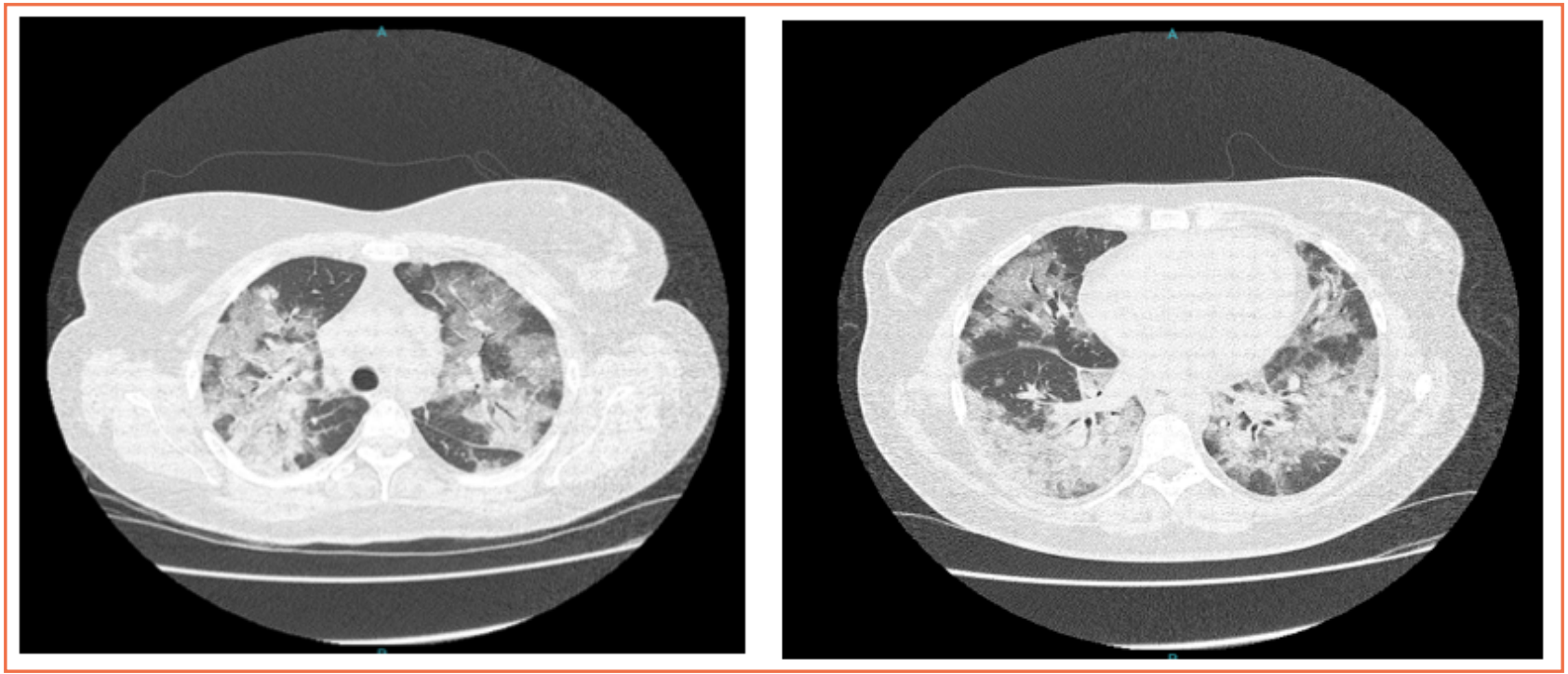

Figure 1. Characteristic pulmonary involvement evidenced in more than $50 \%$ of parenchyma during initial investigation. 
Table 1. Complementary general and lupus activity tests in evolutionary analysis.

\begin{tabular}{cccc}
\hline Complementary tests & Admission & Hospital discharge & Follow-up (60 days) \\
\hline Hemoglobin & 13.8 & 11.5 & 13.2 \\
\hline Overall white blood cells count & 14,500 & 14,700 & 8,400 \\
\hline Lymphocytes $($ absolute $)$ & 900 & 1,980 & 1,480 \\
\hline Platelet count $\left(\times 10^{3}\right)$ & 272,000 & 301,000 & 338,000 \\
\hline Creatinine $(\mathrm{mg} / \mathrm{dL})$ & 0.6 & 0.4 & 0.6 \\
\hline CRP $(\mathrm{mg} / \mathrm{dL})$ & 28.8 & 4.0 & 0.6 \\
\hline Erythrocyte sedimentation rate $(\mathrm{mm} / \mathrm{h})$ & 96 & - & 8 \\
\hline Albumin $(\mathrm{mg} / \mathrm{dL})$ & 3.8 & 3.0 & - \\
\hline Dimer-D $(\mathrm{ng} / \mathrm{mL})$ & 6,934 & 1,844 & - \\
\hline Fibrinogen $(\mathrm{mg} / \mathrm{dL})$ & 86 & 790 & - \\
\hline Proteinuria $(\mathrm{spot}-\mathrm{mg} / \mathrm{dL})$ & 490 & - & 310 \\
\hline $\mathrm{C} 3(\mathrm{mg} / \mathrm{dL})$ & 105 & - & 83.5 \\
\hline C4 $(\mathrm{mg} / \mathrm{dL})$ & 16.1 & - & 21.5 \\
\hline Anti-DNAds $(\mathrm{U} / \mathrm{mL})$ & 49 & & 21.8 \\
\hline
\end{tabular}

\section{CONCLUSION}

We presented a case of a young female SLE patient, without clinical activities or immunosuppressants use, evolving to a severe form of COVID-19; however, with a favorable outcome. The case reinforces the need for more studies and better comprehension about the relationship between autoimmune diseases and the risks for the SARS-CoV-2 infection and prognosis. 